\title{
Author Correction: From genes to drugs: targeted strategies for melanoma
}

\author{
Keith T. Flaherty, F. Stephen Hodi and David E. Fisher
}

Nature Reviews Cancer (2012) https://doi.org/10.1038/nrc3218 Published online 5 April 2012

Several sections of the review should have given attribution to previously published reviews written by the authors.

In the sub-section entitled 'KIT' (page 350 in the PDF), the sentences in the last paragraph beginning with "The current clinical trials..." should have read as follows and cited the reference Ko and Fisher 2011 (REF. $\left.{ }^{194}\right)$ :

As we have discussed previously ${ }^{194}$, the current clinical trials will help to assess whether differential sensitivities among the various KIT-targeting agents exist or correlate with particular KIT mutations. Potential mechanisms of resistance are also being actively investigated. In gastrointestinal stromal tumours (GISTs), the acquisition of additional KIT mutations is a common mechanism of resistance to KIT-targeted drugs $^{30}$. Whether this is true for KIT-mutant melanomas, or whether additional mechanisms are involved, such as amplification or the activation of alternative signalling factors, needs to be determined. There remains great enthusiasm for targeting KIT in mucosal, acral and chronically sun-damaged melanoma subtypes, as KIT is a known oncogene with validated inhibitors ${ }^{194}$.

In the sub-section entitled 'PI3K pathway' (page 355 in the PDF) several sentences should have read as follows and cited Flaherty and Smalley 2008 (REF. ${ }^{195}$ ).

- The penultimate sentence of the first paragraph should read: "The independent therapeutic value of inhibiting the PI3K pathway in melanoma has not been well established, but a substantial body of preclinical evidence supports targeting this pathway as an important adjunct to MAPK pathway-targeted therapy, as we have suggested in a previous review ${ }^{195}$."

- The first sentence of the second paragraph should include a citation: "The optimal point of therapeutic intervention in this pathway is unclear ${ }^{195}$."

- The penultimate sentence of the second paragraph should read "Although the MAPK pathway might represent an essential point of intervention in the treatment of melanoma, as we have noted before, it is crucial that the potential relevance of blocking the PI3K pathway in conjunction with other therapeutic interventions is not overlooked ${ }^{195}$."

In the Conclusions section (page 358 in the PDF), the sentence in the section beginning with "Agents that exhibit significant activity..." should have read as follows and cited REF. ${ }^{195}$ :

"We continue to advocate that agents that exhibit significant activity should be tested in earlier stage patients in order to prevent, rather than treat, metastatic disease ${ }^{195}$."

References

194. Ko, J. M. \& Fisher, D. E. A new era: melanoma genetics and therapeutics. J. Pathol. 223, 241-50 (2011).

195. Flaherty, K. \& Smalley, K. S. M. Somatic genetics and targeted therapies for cutaneous melanoma. Update Cancer Ther. 3, 81-87 (2008).

Note that reference numbering is continued from the list in the original article; for details of reference 30 , please refer to the original article.

https://doi.org/10.1038/s41568-020-00311-3 I Published online 12 October 2020

(C) Springer Nature Limited 2020 